\title{
Evaluation of the Johne's disease risk assessment and management plan on dairy farms in Ontario, Canada
}

\author{
Laura Pieper, ${ }^{*}$ Ulrike S. Sorge,† Trevor J. DeVries,‡ Ann Godkin,§ Kerry Lissemore, ${ }^{*}$ and David F. Kelton*1 \\ *Department of Population Medicine, University of Guelph, Guelph, Ontario, Canada, N1G 2W1 \\ †Department of Veterinary Population Medicine, University of Minnesota, St. Paul 55108 \\ ‡Department of Animal and Poultry Science, University of Guelph, Guelph, Ontario, Canada, N1G 2W1 \\ §Veterinary Science and Policy Group, Ontario Ministry of Agriculture and Food/Ministry of Rural Affairs (OMAF/MRA), Elora, Ontario, \\ Canada, N1G 4Y2
}

\section{ABSTRACT}

Johne's disease (JD) is a production-limiting gastrointestinal disease in cattle. To minimize the effects of JD, the Ontario dairy industry launched the Ontario Johne's Education and Management Assistance Program in 2010. As part of the program, trained veterinarians conducted a risk assessment and management plan (RAMP), an on-farm questionnaire where high RAMP scores are associated with high risk of JD transmission. Subsequently, veterinarians recommended farm-specific management practices for JD prevention. Milk or serum ELISA results from the milking herd were used to determine the herd ELISA status (HES) and withinherd prevalence. After $3.5 \mathrm{yr}$ of implementation of the program, the aim of this study was to evaluate the associations among RAMP scores, HES, and recommendations. Data from 2,103 herds were available for the analyses. A zero-inflated negative binomial model for the prediction of the number of ELISA-positive animals per farm was built. The model included individual RAMP questions about purchasing animals in the logistic portion, indicating risks for between-herd transmission, and purchasing bulls, birth of calves outside the designated calving area, colostrum and milk feeding management, and adult cow environmental hygiene in the negative binomial portion, indicating risk factors for within-herd transmission. However, farms which fed low-risk milk compared with milk replacer had fewer seropositive animals. The model additionally included the JD herd history in the negative binomial and the logistic portion, indicating that herds with a JD herd history were more likely to have at least 1 positive animal and to have a higher number of positive animals. Generally, a positive association was noted between RAMP scores and the odds of receiving a recommendation for the respective risk area; however,

Received September 3, 2014.

Accepted June 17, 2015.

${ }^{1}$ Corresponding author: dkelton@uoguelph.ca the relationship was not always linear. For general JD risk and calving area risk, seropositive herds had higher odds of receiving recommendations compared with seronegative herds if the section scores were low. This study suggests that the RAMP is a valuable tool to assess the risk for JD transmission within and between herds and to determine farm-specific recommendations for JD prevention.

Key words: paratuberculosis, risk assessment, control program, evaluation

\section{INTRODUCTION}

In many countries worldwide, Johne's disease (JD) has been acknowledged as a production-limiting, infectious, gastrointestinal disease in dairy cattle. The disease is caused by Mycobacterium avium ssp. paratuberculosis (MAP), a very slow-growing and intracellular bacterium with a wide host spectrum. In Canada, economic losses due to JD have been estimated at Can $\$ 1,196$ per 100 cows for the entire dairy industry, and Can $\$ 2,992$ in an average-sized, seropositive dairy herd (Tiwari et al., 2008). Losses are attributable to a drop in milk yield, reduced slaughter weight, and early culling. Furthermore, MAP has been associated with Crohn's disease, a chronic gastrointestinal disease in humans. However, evidence supporting a zoonotic potential for MAP is not very strong and a definitive link between MAP and Crohn's disease has yet to be established (Waddell et al., 2008).

To limit the effects of JD on the cattle industry, many countries, including Canada, have implemented voluntary control and surveillance programs. Contrary to many other diseases in which control programs focus on testing and culling of infected animals, JD control is most commonly based on identification and reduction of transmission risk. A MAP infection is not easily detected clinically or with available diagnostic tests, and infected animals can remain undetected for years. Whereas calves have the highest probability of getting infected (Windsor and Whittington, 2010), the highest 
probability of being detected is in older animals. Detection by fecal culture is greatest at an age between 2.5 and $5.5 \mathrm{yr}$, and by antibody titers at an age between 2.5 and $4.5 \mathrm{yr}$ (Nielsen and Ersbøll, 2006). When compared with fecal culture, the sensitivity of JD ELISA in serum or milk has been estimated to be 73.6 and $61.1 \%$, respectively (Hendrick et al., 2005). Therefore, although a traditional test-and-cull program is unlikely to be successful, the tests can help to identify some infected and infectious animals in the herd. A major focus of JD-control programs is on preventing the spread of the disease in the early life of calves through best management practices, many of which are aimed at minimizing the exposure of newborn calves to infectious cows as well as their colostrum, milk, and manure.

A modeling study by Kudahl et al. (2008) demonstrated that although the prevalence of JD would increase when using a test-and-cull program only, it would decrease when combining the test-and-cull program with improved management over the first 6 mo of a calf's life. Furthermore, an empirical study from Canada showed that farms could reduce their JD prevalence through participation in a formal risk assessmentbased JD-control program (Sorge et al., 2011).

In Ontario, Canada, the Ontario Johne's Education and Management Assistant Program (OJEMAP) was piloted in 2006 and launched in January 2010. The program is explained in greater detail on the program website (www.johnes.ca) and in Pieper et al. (2015). Briefly, all Ontario dairy farms were encouraged to do a risk assessment and management plan (RAMP) with their herd veterinarian. To administer the RAMP, trained and registered local veterinarians visited the farm and conducted a risk assessment using a standardized 1-page questionnaire. A detailed guide for the RAMP was provided to ensure objectivity of the assessment. The RAMP was adapted from the risk assessment used by Sorge et al. (2011), but the considerably shortened version used in the program had not yet been formally evaluated since the program was launched.

The RAMP consisted of 5 sections focusing on (1) general JD risk, (2) calving area risk, (3) preweaned heifer risk, (4) postweaned heifer risk, and (5) adult cow risk. Each section was assigned a score, with high-risk scores indicating a high risk of disease transmission. Based on information acquired while administering this questionnaire, veterinarians provided up to 3 farmspecific recommendations for improved JD prevention and control.

Each dairy farm in Ontario was eligible to test all milking cows in the herd for JD via milk or blood ELISA. The costs of the herd test were covered by the program if certain requirements were met. The 2 requirements to be met were: (1) a RAMP had to be conducted within $90 \mathrm{~d}$ of testing and (2) cows that had a high-positive test result [milk optical density (OD) $\geq 1.0$ or serum sample-to-positive ratio $(\mathbf{S}: \mathbf{P}) \geq 1.0]$ were disposed of within $90 \mathrm{~d}$ after the test or before the next calving, whichever came first, while ensuring that the animal did not enter the human food chain and was not sold to another dairy farm. Using all available data from the first $3.5 \mathrm{yr}$ of the OJEMAP, the objectives of our study were to (1) analyze whether RAMP questions identify risk factors for the number of seropositive cows in a herd, and (2) determine whether RAMP scores of the different sections influence recommendations and whether this relationship depends on herd ELISA status (HES).

\section{MATERIALS AND METHODS}

The OJEMAP database, containing observations from 2,103 herds evaluated between January 2010 and August 2013 was used for the analysis. Serum or milk ELISA results from the milking herd, individual questions, sections, and the overall RAMP scores, as well as JD-control recommendations for each herd, were available. The HES was considered positive if at least 1 cow tested positive on the ELISA and was considered negative if all tested animals were ELISA negative. For further data-handling procedures and descriptive statistics of the data, see Pieper et al. (2015). Spearman's rank correlation was used to evaluate associations among RAMP section scores.

All statistical analyses were conducted using the computer program Stata/IC 13.0 for Windows (StataCorp LP, College Station, TX). A probability of $P<$ 0.05 was considered significant.

As shown by Pieper et al. (2015), significant clustering of the RAMP scores were observed at the level of the veterinarian, suggesting that veterinarians had specific management areas that they preferred to focus on. Therefore, clustering by veterinarian was accounted for in the statistical analysis by using mixed logistic regression models and by clustered sandwich estimates for the zero-inflated negative binomial model (ZINB). For graphical display of models addressing the presence of recommendations for a management section, the same fixed effects models that were built using mixed logistic regression were built again using simple logistic regression with clustered sandwich estimates.

For the number of ELISA-positive animals per farm as the outcome, a ZINB model was chosen based on the assumption that a zero count in a herd could arise from 2 different processes: (1) a herd has management practices that prevent it from introducing the infection into the herd, and (2) there are risk factors (management practices) that could reduce the prevalence within a 
herd so that the diagnostic tests are unable to detect infected animals at the time of sampling. The inflated portion of the model is interpreted as the odds of having a zero count, whereas the negative binomial portion is interpreted as a count ratio (CR; Tiwari et al., 2009). A negative coefficient in the inflated portion would therefore indicate an increased odds of having a nonzero count or, in other words, of being an HES-positive farm. In the negative binomial portion, a $\mathrm{CR}>1$ indicates an increase in the number of positive animals on the farm proportional to herds that do not have that risk factor. The model was built by manual forward selection using $P<0.05$ for the RAMP questions as categorical variables and the log-transformed number of tested animals per farm (LNNOTEST) as a linear predictor variable if it had a $P<0.1$ at the univariable level. The variable LNNOTEST was included as an offset in the model. As an approximation for herd size and also for herd density, LNNOTEST was additionally offered to the model in the logistic and negative binomial portion as a confounder for herd management variables. Herd size may be an approximation for herd density based on the hypothesis by Godkin (2011), where, in a bigger herd, a greater number of calves could be exposed to a potentially infectious animal at the time of birth than in a smaller herd. In a smaller herd, fewer cows are calving at a particular time, which minimizes the exposure of a calf to adult cows other than their own dam, even in herds using a group calving pen. The inflated portion of the model was modeled considering only section 1 variables, which consisted of measures of herd biosecurity, introducing JD into the herd, and JD herd history, in addition to LNNOTEST. For the negative binomial portion of the model, all variables from every section of the RAMP were considered. The question "Calving of JD-positive cows in the calving area?" was not offered to the model because the scores were given based on the known within-herd ELISA-positive prevalence of the herd and the cause-effect relationship is reverse for this variable. Only biologically plausible interactions were tested. Different models were compared using the Akaike information criteria and models with lower values were considered superior. The residuals of the final model were calculated by subtracting the predicted from the observed number of positive animals per farm and were graphically assessed to identify potential outliers. The Vuong test and likelihood ratio test were used to determine superiority of the model compared with a regular negative binomial model or a zero-inflated Poisson model, respectively (Dohoo et al., 2009). The predicted and observed probabilities of ELISA-negative or number of ELISA test-positive cows were calculated and displayed (Long and Freese, 2006).
Each of the management recommendations that were given to the farmers after the RAMP had been completed were assigned 1 of 68 different numerical codes by a single person and stored in the program database. For our study, the recommendations were manually associated with the respective questions and sections of the RAMP, based on where the management change would be expected to influence the risk of JD transmission. For the purposes of the data analysis, only the presence ( $\geq 1$ recommendation) or absence (no recommendation) of a recommendation for each management section was considered; additional recommendations for 1 herd that addressed a single section were ignored. Univariable or multivariable mixed logistic regression models with presence of recommendations for each of the 5 management sections as the outcome variables and RAMP section scores as explanatory variables were built with veterinarian as a random effect. The linearity of the relationships was assessed by locally weighted scatterplot smoothing (lowess) and quadratic terms were offered for sections 3 and 5 . Section 1 score was categorized $(0$ or $>1)$ to account for the bimodal distribution, with many herds having a zero value, thus indicating that they did not introduce animals into their herd. The HES was tested as a confounding variable for the relationship between RAMP scores and the presence of recommendations for a management area. Confounding variables were only retained if the change in the $\beta$-coefficient of the RAMP section score was $>30 \%$, regardless if the confounding variable was significant in the model or not. The intraclass correlation coefficient was calculated by the latent variable technique (Dohoo et al., 2009).

\section{RESULTS}

Overall, $27.2 \%$ of herds had at least 1 ELISA-positive animal and the within-herd prevalence was $0.85 \%(95 \%$ $\mathrm{CI}=0.79-0.94 \%)$ in all and $3.2 \%(95 \% \mathrm{CI}=2.9-3.4 \%)$ in seropositive herds. No associations were found between a section score of 1 and scores for sections 3, 4 , and 5 , respectively. The remaining correlations were positive, with the lowest values between section scores 1 and $2(\mathrm{r}=0.09, P<0.001)$ and highest values between section scores 2 and $5(\mathrm{r}=0.40 ; P<0.001)$. Veterinarians gave $56,79,54,25$, and $8 \%$ of farmers' recommendations for section $1,2,3,4$, and 5 , respectively.

Figure 1 shows the observed and predicted probability of the number of ELISA-positive animals per farm (upper panel) and the difference between observed and predicted probability of the number of ELISA-positive animals per farm (lower panel) based on the ZINB model. The predicted and observed probabilities (Fig- 
ure 1, upper panel) are overlapping, indicating good model predictions for the model. The model slightly overestimates the probability of zero counts, underestimates the probability of farms having 1 test-positive cow, and again overestimates the probability of farms having 2 to 7 ELISA-positive animals (Figure 1, lower panel). The Vuong test $(\mathrm{z}=4.80, P<0.001)$ and the likelihood ratio test $\left(\chi^{2}=284.5, P<0.001\right)$ indicated the superiority of the ZINB model compared with regular negative binomial model or zero-inflated Poisson model, respectively. Herds were less likely to have a zero count of ELISA-positive cows if they were larger herds compared with smaller herds and purchased animals from multiple herds compared with not purchasing animals (Table 1). Additionally, the odds of having no ELISA-positive animals were greatly reduced for farms that had previously detected JD clinical or test-positive animals in the herd. Herds were predicted to have more ELISA-positive cows if they had a history of JD clinical or test-positive animals, if more than $10 \%$ of calves were born outside of the designated calving area, if they purchased a bull in the last $5 \mathrm{yr}$, if they fed high-risk colostrum, and if the hygienic condition of the milking cow environment was poor. Farms were predicted to have fewer positive cows if calves were fed low-risk whole milk compared with milk replacer; but no difference was noted between feeding milk replacer and feeding whole milk from cows without selection for JD or from the bulk tank. An interaction term between the milk and colostrum feeding variables (section 3, question 1 and section 3, question 3) was not significant.

Table 2 and Figure 2 demonstrate the relationships between the RAMP section score and receiving a recommendation for that management area. Generally, a positive association was seen between section scores and receiving a recommendation; however, the relationship was curvilinear for sections 3 and 5 due to the introduction of a quadratic term. For section 3, although the model predicts a decreasing probability of receiving a recommendation after a score peak at 37 points, the confidence intervals get wider and the upper bound continues to predict a slowly increasing probability. The probability of receiving a recommendation for sections 1, 2, and 5 was influenced by HES. An interaction was present for the relationship between receiving a recommendation for general JD risk management and section 1 score, as well as receiving a recommendation for calving area risk management and section 2 score. In both cases, farms with a low section score and a negative HES were less likely to receive a recommendation compared with farms with a low section score and a positive HES. The probability of receiving a recommendation was not different for HES-positive or -negative
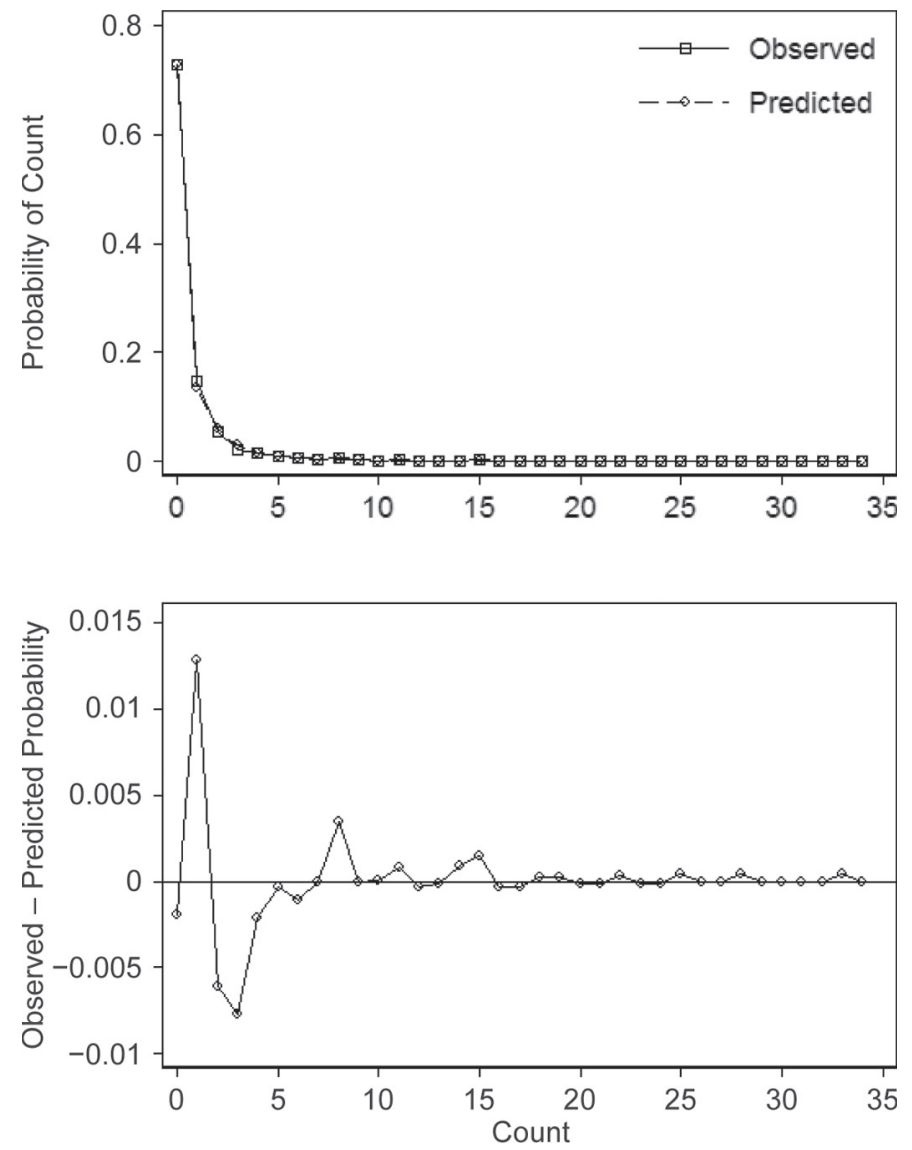

Figure 1. Prediction from a zero-inflated negative binomial model: observed and predicted probability of count of ELISA positive cows per herd (upper panel); difference between observed and predicted probability of count of ELISA positive animals per farm (lower panel).

farms if they received high section 1 and 2 scores. For section 5, HES negatively influenced the probability of receiving a recommendation, indicating that producers would be less likely to receive recommendations if their HES was positive. It is noteworthy that the level of the predicted probability of receiving a recommendation varied substantially, from approaching 100\% for section 2 (Figure $2 \mathrm{~b}$ ) to $<24 \%$ for section 5 (Figure $2 \mathrm{e}$ ). Concurrently, between 15 (section 3 ) and $39 \%$ (section 1) of the variability of receiving a recommendation was explained by the assessing veterinarian (Table 2).

\section{DISCUSSION}

In our study, associations among the RAMP scores, herd ELISA results, and RAMP recommendations were investigated. In the ZINB model, purchasing animals from multiple herds was found to be a risk factor for being an ELISA-positive herd. This is consistent with previous reports in which purchasing animals was associated with a positive herd status (Wells and Wagner, 
Table 1. Zero-inflated negative binomial model predicting the number of ELISA-positive animals per herd $(\mathrm{n}=2,103)$ using risk assessment and management plan questions

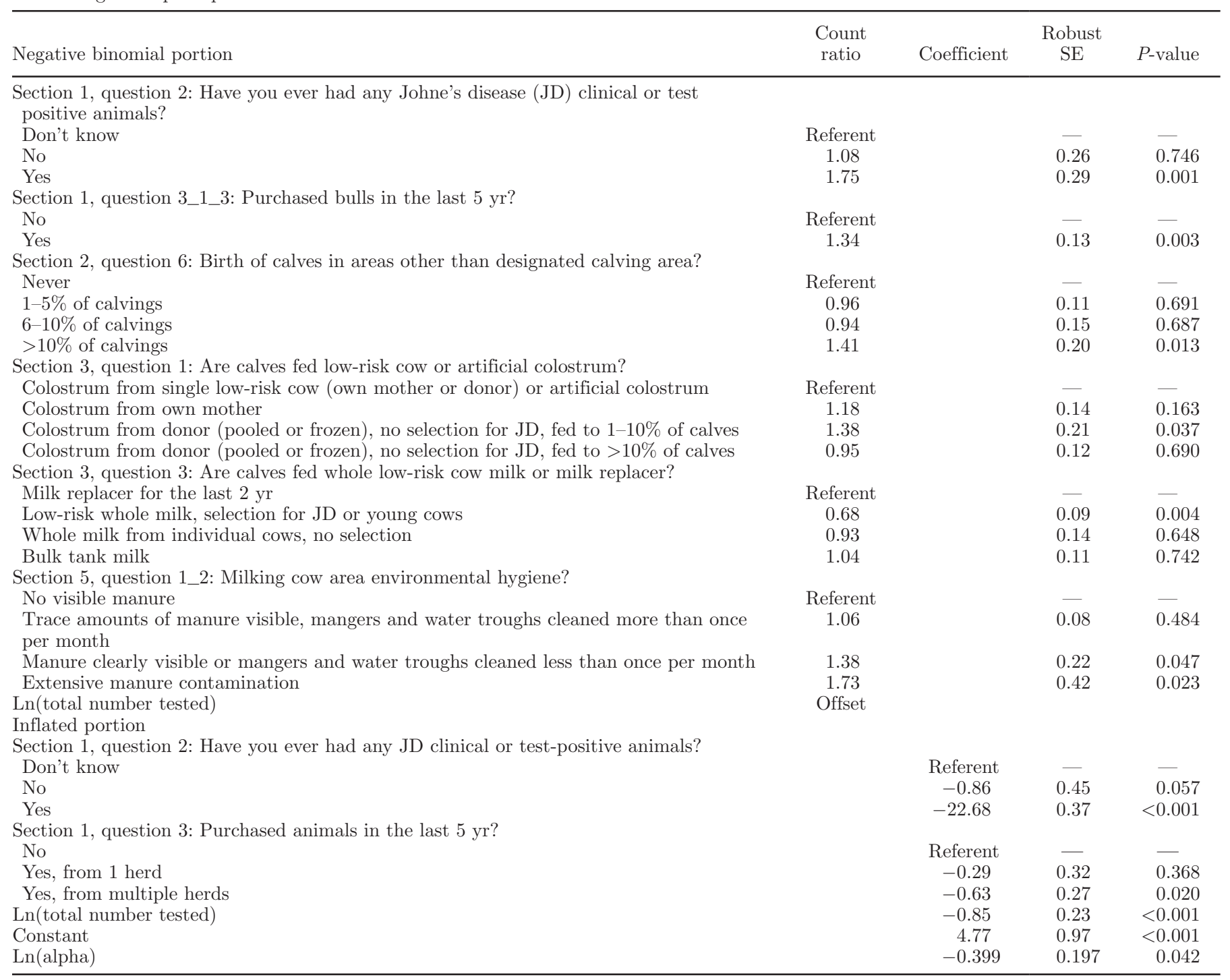

2000; Sorge et al., 2012). However, purchasing animals from 1 herd was not associated with the HES. It might be that producers that purchased from 1 herd only knew the herd and the herd's disease status and therefore purchased low-risk animals compared with producers that purchased from multiple herds.

After accounting for purchasing animals within the last $5 \mathrm{yr}$ in the logistic portion of the model, questions about purchasing cows or heifers were no longer significant and only purchasing bulls remained significant in the negative binomial portion of the model. This might indicate that purchasing bulls was associated with additional risks that are not included in just bringing in animals from other herds. It is unclear whether these bulls were used for breeding purposes or were simply beef bulls on the farm. Previously, MAP has been isolated from sperm and reproductive organs of breeding bulls (Khol et al., 2010), but transmission of MAP through mating has not yet been reported. Contrarily, Tiwari et al. (2009) described a JD ELISA-positive CR $<1$ if the farms purchased bulls within the last year; those authors suggested that it was a surrogate for an unmeasured herd management variable.

Consistent with previous reports (Wells and Wagner, 2000; Muskens et al., 2003), herd size was a significant risk factor for having at least 1 ELISA-positive animal in the herd. One might argue that due to the imperfect specificity of the test, a small number of animals might test positive, even though they are truly noninfected or negative (false positive). Conversely, due to the low sensitivity of the test, smaller herds might be falsely identified as negative. Considering that the herd sensitivity 
and specificity are dependent on the herd size, herds might be more likely to have at least 1 test-positive cow, the cut-off for HES in our study, if more animals were tested (Dohoo et al., 2009). Beyond being merely an effect of herd sensitivity and specificity, herd size could have further affected the probability of a herd having a nonzero count. Herd size could be a measure of herd density at calving, as outlined earlier (Godkin, 2011). Thus, in a larger herd, cow density in the calving area might be higher and potentially infectious contacts and environmental contamination could be more likely.

Farms were predicted to have fewer ELISA-positive animals if they fed low-risk colostrum or artificial colostrum compared with feeding colostrum from the dam or a donor cow without selection for JD. This is consistent with Pithua et al. (2009) and Sorge et al., (2012).

A surprising result of the model was that herds had fewer ELISA-positive cows if higher-risk practices were recorded for milk-feeding management. The present study is based on a cross-sectional design and causal inferences of the observed associations cannot be drawn. One might even speculate that reverse causation could have occurred in cases where farmers knew their JD status and changed their management accordingly in the desired manner (e.g., feeding milk replacer instead of pooled milk) to reduce the risk of disease transmission on their farm.

Poor milking cow area environmental hygiene was a risk factor for a greater number of ELISA-positive cows in the herd. It might be that an unhygienic environment contributes to an infection of adult animals as they enter the milking herd. As the animals get older it is more difficult, but not impossible, for them to get infected with JD. Windsor and Whittington (2010) showed that only $19 \%$ of animals infected after 12 mo develop a detectable infection and even less develop signs of clinical JD. Several studies summarized by the aforementioned authors reported detection of MAP by culture in intestines or lymph nodes in animals infected at age $>24$ mo. Similarly, a recent observational study confirmed that infection of adult, MAP-unexposed cows was possible after introduction into a JD-positive herd (Espejo et al., 2013). Consequently, environmental contamination through actively shedding animals might contribute to a higher within-herd JD prevalence in the milking herd.

Previous reports have also included the JD herd history in model predictions (Johnson-Ifearulundu and Kaneene, 1998; Tavornpanich et al., 2008; Sorge et al., 2012). A strong positive association was noted between the JD herd history and the HES, as well as the within-herd prevalence in our study, indicating that this is likely valuable information for potential buyers of animals from this herd.

Usually, veterinarians conducted the RAMP after receiving the ELISA results. Hence, they were not blinded toward the herd status and might have given higher scores to HES-positive farms. This could have

Table 2. Mixed logistic regression analyses investigating the association between receiving a recommendation for a specific management area and the risk assessment and management plan score for that section and herd ELISA status (HES)

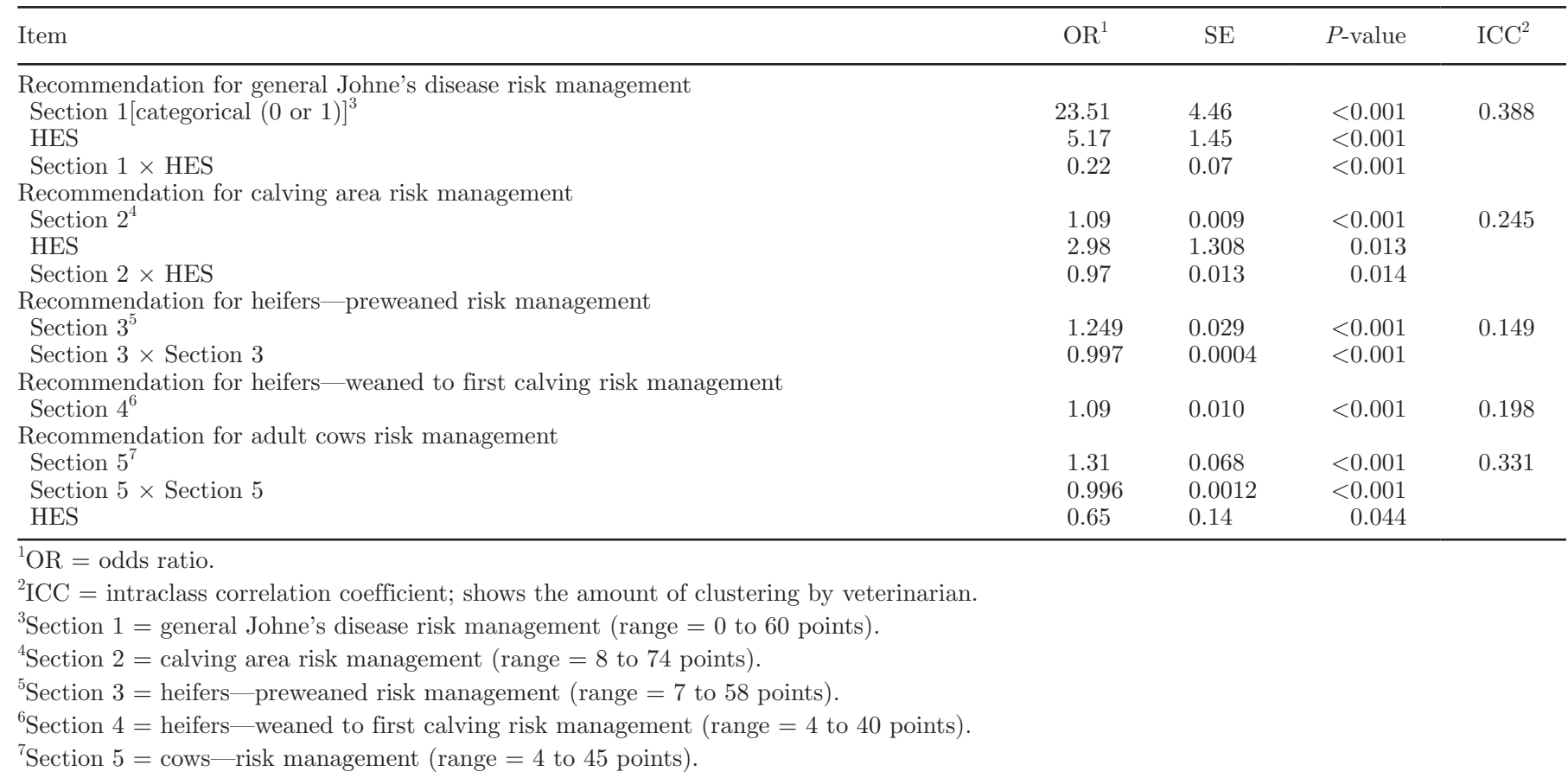



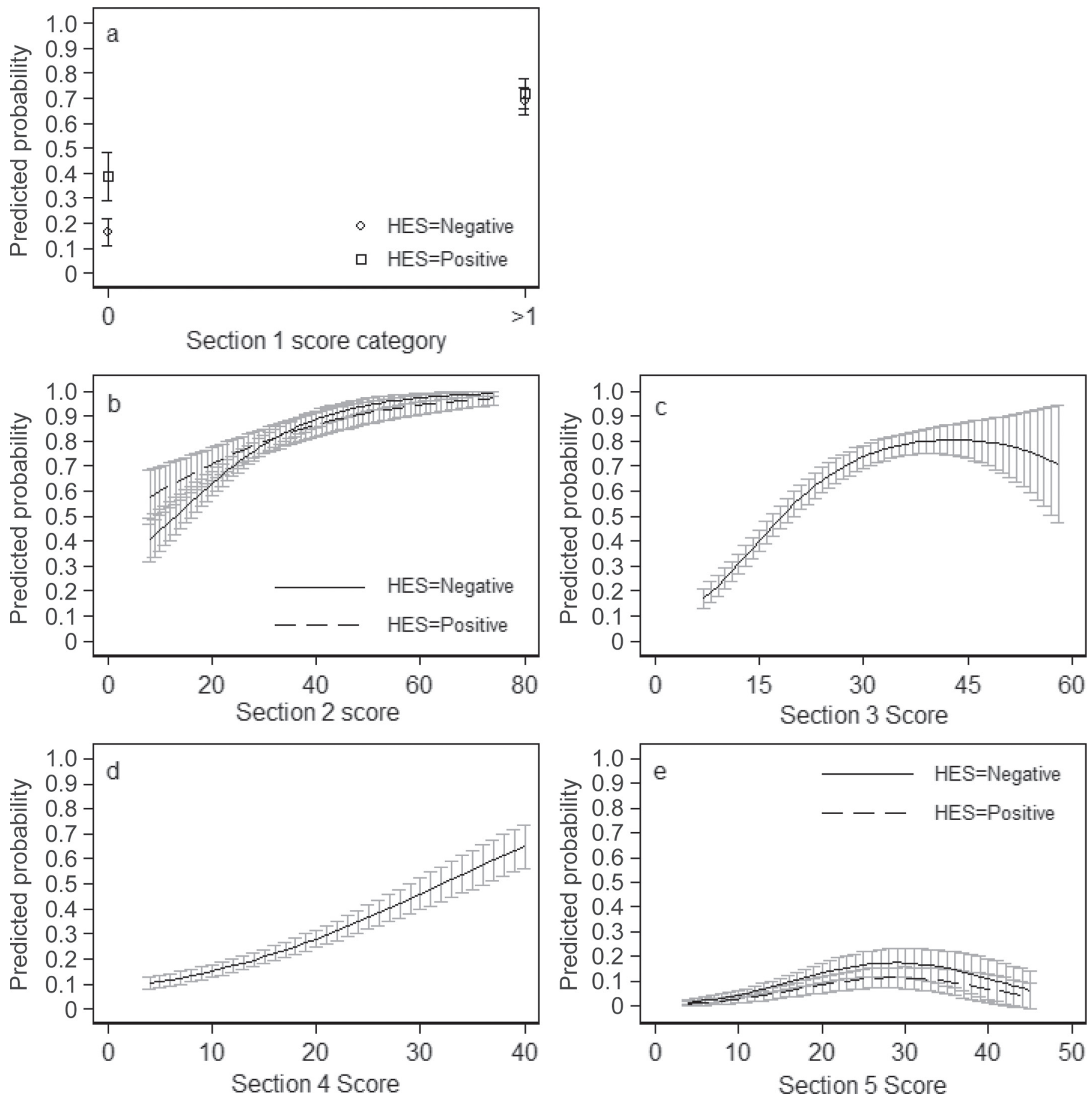

Figure 2. Predicted probability of receiving a recommendation for a risk assessment and management plan (RAMP) section by respective RAMP section score and Johne's disease herd ELISA status (HES); (a) recommendation for general Johne's disease risk management, (b) recommendation for calving area risk management, (c) recommendation for preweaned heifer risk management, (d) recommendation for weaned heifer to first calving risk management, and (e) recommendation for adult cow risk management. X-scales were adjusted according to the range of the section scores. Error bars indicate $95 \%$ CI.

increased the observed measures of association and, therefore, biased the results of our study.

Not all questions were associated with the HES or number of ELISA-positive animals; it would therefore be possible to remove some questions from the RAMP. However, there might be use in retaining those additional questions, despite the lack of evidence for their relevance for JD control. The questions provide a valu- 
able opportunity for the veterinarian to inspect management areas that are not commonly considered during herd health visits and discuss management strategies with the producer (Sorge et al., 2010). Furthermore, the relative importance of management areas for JD control was accounted for by increasing or decreasing the number of questions for the sections. For example, the calving section had a total of 8 questions and the preweaned calf rearing section had 7 questions, whereas the postweaning calf rearing section only contained 4 questions.

Moreover, the strength of the relationship between the answer categories of a question and the herd ELISA prevalence might not be reflected in the assigned RAMP question scores. The relationships are rarely linear and the RAMP question scores might be misleading in this regard. Thus, when using epidemiological models to describe the relationship between ELISA prevalence and herd management variables with categorical responses, the variables should be offered using the categorical description as opposed to continuous variables using the assigned numerical question scores.

The relationship between the RAMP section scores and receiving a recommendation was positive. Therefore, herds were more likely to receive a recommendation for a specific management area if they had received a higher score for this area. The relationship was not linear for sections 3 and 5 . This indicates that, after the turning point, the probability of receiving a recommendation was not further increasing or even decreasing with increasing section score. For section 3, predictions for high section scores might not be very precise due to the sparse number of observations in this area. For section 5, a generally low probability of receiving a recommendation was noted, and farms with high scores also had high scores in another area (e.g., section 2), causing the veterinarian to give recommendations in the other area. Further, for section 5 the probability was even lower for HES-positive than for -negative herds. It might be that veterinarians focused their attention on management changes in other areas (sections 1 and 2) with a more clearly demonstrated link to JD prevention. Therefore, HES-positive farms may not have been more, or even less, likely to receive a recommendation for that area. Furthermore, a great amount of variability in the probability of receiving a recommendation was explained by the assessing veterinarian. This might further indicate that differences exist in veterinarians' preferences in giving recommendations for specific management areas, which might be influenced by many factors including the veterinarians' knowledge or experience and their perceptions of an individual farmer's management goals or priorities (Derks et al. 2013). To the authors' knowledge, this is the first study investigating the relationship between risk scores for JD transmission and the probability of receiving a recommendation for a management area.

\section{CONCLUSIONS}

Positive associations were observed between the number of ELISA-positive animals per herd and RAMP questions. Purchasing animals from multiple herds was a strong risk factor for being a HES-positive farm; however, management practices associated with colostrum management and environmental hygiene were associated with having more animals testing positive on the farm. The definitive role of purchasing bulls in having more positive animals on the farm is still unknown. Overall, the RAMP is a useful tool for the veterinarian and producer for identifying recommendations for management improvement for JD prevention on the farm.

\section{ACKNOWLEDGMENTS}

This research was kindly funded by the University of Guelph-Ontario Ministry of Agriculture and Food (OMAF) research partnership. The authors sincerely thank Nicole Perkins and Jamie Imada (University of Guelph) for their work and dedication to the OJEMAP. Nicole Perkins coordinated the OJEMAP and Jamie Imada coded all recommendations into numerical codes. Without their help, it would have been difficult to conduct the program smoothly and successfully.

\section{REFERENCES}

Derks, M., B. van Woudenbergh, M. Boender, W. Kremer, T. van Werven, and H. Hogeveen. 2013. Veterinarian awareness of farmer goals and attitudes to herd health management in The Netherlands. Vet. J. 198:224-228.

Dohoo, I. R., W. Martin, and H. Stryhn. 2009. Veterinary Epidemiologic Research. 2nd ed. VER Inc., Charlottetown, Canada.

Espejo, L. A., N. Kubat, S. M. Godden, and S. J. Wells. 2013. Effect of delayed exposure of cattle to Mycobacterium avium ssp. paratuberculosis on the development of subclinical and clinical Johne's disease. Am. J. Vet. Res. 74:1304-1310.

Godkin, A. 2011. Johne's disease: The Ontario program-Experiences from 2005 to 2011. Pages 73-80 in Western Canadian Dairy Seminar, Advances in Dairy Technology, Edmonton, Alberta, Canada. Department of Agricultural, Food \& Nutritional Science, University of Alberta, Edmonton, Canada.

Hendrick, S. H., T. Duffield, D. Kelton, K. Leslie, K. Lissemore, and M. Archambault. 2005. Evaluation of enzyme-linked immunosorbent assays performed on milk and serum samples for detection of paratuberculosis in lactating dairy cows. J. Am. Vet. Med. Assoc. 226:424-428.

Johnson-Ifearulundu, Y. J., and J. B. Kaneene. 1998. Managementrelated risk factors for $M$. paratuberculosis infection in Michigan, USA, dairy herds. Prev. Vet. Med. 37:41-54.

Khol, J. L., P. Kralik, I. Slana, V. Beran, C. Aurich, W. Baumgartner, and I. Pavlik. 2010. Consecutive excretion of Mycobacterium avium subspecies paratuberculosis in semen of a breeding bull compared to the distribution in feces, tissue and blood by IS900 and 
F57 quantitative real-time PCR and culture examinations. J. Vet. Med. Sci. 72:1283-1288.

Kudahl, A. B., S. S. Nielsen, and S. Østergaard. 2008. Economy, efficacy, and feasibility of a risk-based control program against paratuberculosis. J. Dairy Sci. 91:4599-4609.

Long, J. S., and J. Freese. 2006 Regression Models for Categorical Dependent Variables Using Stata. 2nd ed. Stata Press, College Station, TX.

Muskens, J., A. R. W. Elbers, H. J. Van Weering, and J. P. T. M. Noordhuizen. 2003. Herd management practices associated with paratuberculosis seroprevalence in Dutch dairy herds. J. Vet. Med. B Infect. Dis. Vet. Public Health 50:372-377.

Nielsen, S. S., and A. K. Ersbøll. 2006. Age at occurrence of Mycobacterium avium subspecies paratuberculosis in naturally infected dairy cows. J. Dairy Sci. 89:4557-4566.

Pieper, L., T. J. DeVries, U. S. Sorge, A. Godkin, K. J. Hand, N. R. Perkins, J. Imada, and D. F. Kelton. 2015. Variability in the risk assessment and management plan (RAMP) score completed as part of the Ontario Johne's Education and Management Assistance Program (2010-2013). J. Dairy Sci. 98:2419-2426.

Pithua, P., S. M. Godden, S. J. Wells, and M. J. Oakes. 2009. Efficacy of feeding plasma-derived commercial colostrum replacer for the prevention of transmission of Mycobacterium avium ssp. paratuberculosis in Holstein calves. J. Am. Vet. Med. Assoc. 234:1167-1176.

Sorge, U. S., J. Mount, D. F. Kelton, and A. Godkin. 2010. Veterinarians' perspective on a voluntary Johne's disease prevention program in Ontario and Western Canada. Can. Vet. J. 51:403-405.

Sorge, U. S., K. Lissemore, A. Godkin, J. Jansen, S. Hendrick, S. Wells, and D. F. Kelton. 2011. Changes in management practices and apparent prevalence on Canadian dairy farms participating in a voluntary risk assessment-based Johne's disease control program. J. Dairy Sci. 94:5227-5237.

Sorge, U. S., K. Lissemore, A. Godkin, J. Jansen, S. Hendrick, S. Wells, and D. F. Kelton. 2012. Risk factors for herds to test positive for Mycobacterium avium ssp. paratuberculosis-antibodies with a commercial milk enzyme-linked immunosorbent assay (ELISA) in Ontario and Western Canada. Can. Vet. J. 53:963-970.

Tavornpanich, S., W. O. Johnson, R. J. Anderson, and I. A. Gardner. 2008. Herd characteristics and management practices associated with seroprevalence of Mycobacterium avium ssp. paratuberculosis infection in dairy herds. Am. J. Vet. Res. 69:904-911.

Tiwari, A., J. A. VanLeeuwen, I. R. Dohoo, G. P. Keefe, J. P. Haddad, H. M. Scott, and T. Whiting. 2009. Risk factors associated with Mycobacterium avium subspecies paratuberculosis seropositivity in Canadian dairy cows and herds. Prev. Vet. Med. 88:32-41.

Tiwari, A., J. A. VanLeeuwen, I. R. Dohoo, G. P. Keefe, and A Weersink. 2008. Estimate of the direct production losses in Canadian dairy herds with subclinical Mycobacterium avium subspecies paratuberculosis infection. Can. Vet. J. 49:569-576.

Waddell, L. A., M. Sc, A. Rajic, J. Sargeant, J. Harris, R. Amezcua, L. Downey, S. Read, and S. A. McEwen. 2008. The zoonotic potential of Mycobacterium avium ssp. paratuberculosis: A systematic review. Can. J. Public Health 99:145-155.

Wells, S. J., and B. A. Wagner. 2000. Herd-level risk factors for infection with Mycobacterium paratuberculosis in US dairies and association between familiarity of the herd manager with the disease or prior diagnosis of the disease in that herd and use of preventive measures. J. Am. Vet. Med. Assoc. 219:1450-1457.

Windsor, P. A., and R. J. Whittington. 2010. Evidence for age susceptibility of cattle to Johne's disease. Vet. J. 184:37-44. 